Received: 23.04 .2018

Revised: 07.06.2019

Accepted: 28.06 .2019

DOI: $10.17804 / 2410-9908.2019 .3 .016-024$

\title{
DETERMINING THE PARAMETERS OF A PIPE SYSTEM CONTAINING FLUID FLOW BY NATURAL FREQUENCIES OF FLEXURAL VIBRATIONS
}

\author{
A. G. Khakimov \\ Mavlyutov Institute of Mechanics, UFRC RAS, \\ 71 Oktyabrya Ave., Ufa, 450054, Russian Federation \\ (iD https://orcid.org/0000-0003-4093-5380 @ hakimov@anrb.ru \\ Corresponding author. E-mail: hakimov@anrb.ru \\ Address for correspondence: pr. Oktyabrya, 71, Ufa, 450054, Russian Federation \\ Tel.: +7 (347) 29214 06; fax: +7 (347) 2355255
}

The investigation deals with natural frequencies of flexural vibrations in a pipe system containing pressurized fluid flow and clamped at both ends. It has been found that an increase in the axial load results in higher natural frequencies of pipe flexural vibrations. The research has shown that an increase in the pipe wall thickness results in lower natural frequencies of flexural vibrations under tensile axial loading and higher natural frequencies of flexural vibrations under compressive axial loading. It has also been found that an increase in fluid density or pressure within the pipe system results in lower natural frequencies of pipe flexural vibrations.

Using two natural frequencies of pipe flexural vibrations, we can determine axial load and pipe wall thickness, or axial load and fluid density within the pipe system, or fluid density within the pipe system and pipe wall thickness, or fluid pressure and density within the pipe system, or fluid pressure and pipe wall thickness.

The investigation outcomes can be applied for assessing axial load and pipe wall thickness, or axial load and fluid density within a pipe system, or fluid density within a pipe system and pipe wall thickness, or fluid pressure and density within the pipe system, or fluid pressure and pipe wall thickness using two natural frequencies of flexural vibrations.

Keywords: pipe system, flexural vibrations, natural frequencies, axial load, wall thickness, fluid density, pressure, primal and inverse problems.

\section{Acknowledgments}

The study was financed by the federal budget according to state assignment No. 0246-2019-0088) and supported by RFBR grant No. 18-01-00150.

\section{References}

1. Sidorov B.V., Martynov S.A. Recommended Technology for the Diagnostics of Underground Pipelines. Kontrol. Diagnostika, 2005, no. 12, pp. 18-19. (In Russian).

2. Gladwell G.M.L. Inverse problems in vibration. Dordrecht, Boston, London, Kluwer Academic Publishers, 2004.

3. Guangming Dong, Jin Chen. Vibration analysis and crack identification of a rotor with open cracks. Japan Journal of Industrial and Applied Mathematics, 2011, vol. 28, no. 1, pp. 171-182. DOI: 10.1007/s13160-011-0031-3. 
4. Akop'yan V.A., Cherpakov A.V., Rozhkov E.V., and Solov'ev A.N. Integral indicator for damage identification in rod-shaped structural components. Kontr. Diagnost., 2012, no. 7, pp. 50-56. (In Russian).

5. Yunwei Zhang, Guozheng Yan. Detection of gas pipe wall thickness based on electromagnetic flux leakage. Russian Journal of Nondestructive Testing, 2007, vol. 43, iss. 2, pp 123-132. DOI: $10.1134 /$ S1061830907020088.

6. Kulikov V.V. The thickness of tube's wall in pressure. Stroitelstvo neftyanykh i gazovykh skvazhin na sushe i na more, 2009, no 7, pp. 6-8. (In Russian).

7. Kucheryavyi V.I., Mil'kov S.N. Reliability analysis of a compression section of a gas pipeline with the presence of longitudinal cracks. Journal of Machinery Manufacture and Reliability, 2011, vol. 40, no. 3, p. 290-293. DOI: 10.3103/S1052618811030095.

8. Novgorodov D.V., Rybalko V.G., Shleyenkov A.S. The stress state instability factor and its effect on the growth of stress corrosion cracking defects. Diagnostics, Resource and Mechanics of materials and structures, 2018, iss. 6, pp. 249-254. DOI: 10.17804/2410-9908.2018.6.249-254.

9. Novgorodov D.V., Rybalko V.G., Shleyenkov A.S., and Surkov A.Yu. A study of gas pipeline emergency failure. Diagnostics, Resource and Mechanics of materials and structures, 2018, iss. 6, pp. 237-248. DOI: 10.17804/2410-9908.2018.6.237-248.

10. Chirikov V.A., Dimitrov D.M., Kostov K.P. Universal experimental relation for natural friquencies of transversal vibration of stubby free-free beams. Diagnostics, Resource and Mechanics of materials and structures, 2015, iss. 4, pp. 42-51. DOI: 10.17804/2410-9908.2015.4.042-051.

11. Khakimov A.G. Determination of fluid density and inner pressure in a pipeline using natural frequencies of flexural vibrations. Problemy sbora, podgotovki i transporta nefti i nefteproduktov, 2014, no. 1, pp. 37-43. (In Russian).

12. Prochnost. Ustoichivost. Kolebaniya. Spravochnik. T. 3 [Strength. Stability. Oscillations. Handbook, Birger I.A. and Panovko Ya.G., eds., vol. 3]. Moscow, Mashinostroenie Publ., 1968. (In Russian).

13. Bolotin V.V. Vibratsii v tekhnike. Spravochnik v 6 tomakh. T. 1. Kolebaniya lineinykh sistem [Vibrations in Technology: A Handbook. Vol. 1. Vibrations of Linear Systems]. Moscow, Mashinostroenie Publ., 1999, 504 p. (In Russian). 
Подана в журнал: 23.04.2019

УДК 532.3; 532.5

DOI: $10.17804 / 2410-9908.2019 .3 .016-024$

\title{
ОПРЕДЕЛЕНИЕ ПАРАМЕТРОВ ТРУБОПРОВОДА С ЖИДКОСТЬЮ ПО СОБСТВЕННЫМ ЧАСТОТАМ ИЗГИБНЫХ КОЛЕБАНИЙ
}

\author{
А. Г. Хакимов \\ Институт механики им. Р.Р. Малютова Уфимского федерального исследовательского центра РАН, \\ пр-т Октября, 71, 450054, г. Уфа, Российская Федерация \\ iD https://orcid.org/0000-0003-4093-5380 ه hakimov@anrb.ru \\ Ответственный автор. Электронная почта: hakimov@anrb.ru \\ Адрес для переписки: пр-т Октября, 71, 450054, г. Уфа, Российская Федерация \\ Тел.: +7 (347) 292-14-06; факс: +7 (347) 235-52-55
}

Исследованы собственные частоты изгибных колебаний защемленных по краям трубопровода с жидкостью под давлением. С увеличением осевого усилия происходит увеличение собственных частот изгибных колебаний трубы. Установлено, что с увеличением толщины стенки трубы происходит уменьшение собственных частот изгибных колебаний трубы для растягивающего осевого усилия и увеличение собственных частот изгибных колебаний трубы для сжимающего осевого усилия, а с увеличением плотности жидкости или давления внутри трубопровода происходит уменьшение собственных частот изгибных колебаний трубы.

По двум собственным частотам изгибных колебаний трубы можно определить осевое усилие и толщину стенки трубы или осевое усилие и плотность жидкости в трубопроводе или плотность жидкости в трубопроводе и толщину стенки трубы, или давление и плотность жидкости в трубопроводе, или давление в трубопроводе и толщину его стенки.

Результаты работы могут быть применены для определения осевого усилия и толщины стенки трубы или осевого усилия и плотности жидкости в трубопроводе, или плотности жидкости в трубопроводе и толщины стенки трубы, или давления и плотности жидкости в трубопроводе, или давления в трубопроводе и толщины его стенки по двум собственным частотам изгибных колебаний.

Ключевые слова: трубопровод, изгибные колебания, собственные частоты, осевое усилие, толщина стенки, плотность жидкости, давление, прямая и обратная задачи.

\section{1. Введение}

В протяженных объектах типа магистральных трубопроводных систем не все участки могут быть доступны для визуального осмотра и приборного диагностирования [1]. Проблемам диагностики дефектов в стержневых системах посвящено много работ, например, в [2-4]. В [5] представлен способ неразрушающего контроля толщины стенки газопровода, основанный на методе магнитного поля рассеяния, и дается обзор работ по данной теме. В [6] предложены формы представления известных зависимостей, позволяющие непосредственно рассчитать необходимую толщину стенки труб. В статье [7] разработан алгоритм, позволяющий найти математическое ожидание длины продольной трещины дефектной трубы, при которой сохраняется нормативный безопасный уровень надежности газопровода. Решение получено для условия, когда вязкость разрушения трубной стали, сжимающее усилие, давление газа, коэффициент линейного расширения, модуль Юнга, температурный перепад стенки трубы, ее диаметр и толщина нормально распределены. В [8] выполнен краткий анализ результатов внутритрубной дефектоскопии 
протяженного участка газопровода. В работе [9] приведены результаты исследования причин аварийного разрушения газопровода диаметром 377 мм с толщиной стенки 6 мм для спиральношовной трубы. На основании серии экспериментальных испытаний в работе [10] установлена универсальная экспериментальная зависимость для определения собственных поперечных частот коротких цельных стержней со свободными концами. В [11] определяется плотность жидкости или осевой момент инерции поперечного сечения и внутреннее давление по собственным частотам изгибных колебаний трубопровода. Здесь исследуются собственные частоты изгибных колебаний трубопровода с жидкостью под давлением, находящейся под действием растягивающей силы, а также решена обратная задача определения осевого усилия и толщины стенки трубы или осевого усилия и плотности жидкости в трубопроводе, или плотности жидкости в трубопроводе и толщины стенки трубы, или давления и плотности жидкости в трубопроводе, или давления в трубопроводе и толщины его стенки по двум собственным частотам изгибных колебаний.

\section{2. Постановка задачи}

Исследуются собственные частоты изгибных колебаний трубопровода с жидкостью под давлением, находящейся под действием растягивающей силы и защемленной по краям. Требуется определить осевое усилие и толщину стенки трубы или осевое усилие и плотность жидкости в трубопроводе, или плотность жидкости в трубопроводе и толщину стенки трубы, или давление и плотность жидкости в трубопроводе, или давление в трубопроводе и толщину его стенки по собственным частотам изгибных колебаний.

Уравнение изгибных колебаний трубопровода по модели Кирхгоффа имеет вид:

$$
E J \frac{\partial^{4} w_{*}}{\partial x^{4}}-\left(T-P_{i} F_{i}\right) \frac{\partial^{2} w_{*}}{\partial x^{2}}+\left(\alpha F+\rho_{i} F_{i}\right) \frac{\partial^{2} w_{*}}{\partial t^{2}}=0,
$$

где $E, \rho, J, F$ - модуль упругости, плотность, осевой момент инерции и площадь поперечного сечения трубопровода; $\rho_{i}, F_{i}, P_{i}$ - плотность жидкости, площадь проходного сечения и давление внутри трубопровода; $T$ - усилие растяжения в трубопроводе; $w *-$ прогиб трубопровода; $x$ - координата, направленная по оси трубопровода; $t$ - время. А уравнение, определяющее форму изгибных колебаний трубопровода, имеет вид:

$$
\begin{gathered}
\frac{\partial^{4} w_{*}}{\partial x^{4}}-\frac{T-P_{i} F_{i}}{E J} \cdot \frac{\partial^{2} w_{*}}{\partial x^{2}}-\frac{\left(\alpha F+\rho_{l} F_{i}\right) \omega^{2}}{E J} w_{*}=0 ; \\
F_{i}=\pi R_{i}^{2}, F=\pi\left[\left(R_{i}+h\right)^{2}-R_{i}^{2}\right], J=\pi\left[\left(R_{i}+h\right)^{4}-R_{i}^{4}\right] / 4,
\end{gathered}
$$

где $\omega$ - частота; $R_{i}$ - внутренний радиус трубопровода; $h, L$ - толщина стенки и длина трубопровода. Отсчитывая координату $x$ от точки крепления, запишем граничные условия для защемленного по краям трубопровода:

$$
w_{*}=0, \frac{\partial w_{*}}{\partial x}=0,(x=0, L) .
$$


Пользуясь в дальнейшем обозначениями

$$
\begin{aligned}
& \xi=\frac{x}{L} ; w=\frac{w_{*}}{L} ; p=\frac{\left(T-P_{i} F_{i}\right) L^{2}}{2 E J} ; \lambda=L \sqrt[4]{\frac{\left(\rho+\rho_{i} F_{i}\right) \omega^{2}}{E J}} \\
& \lambda_{\Psi}=\sqrt{\sqrt{p^{2}+\lambda^{4}}-p} ; \quad \lambda_{2}=\sqrt{\sqrt{p^{2}+\lambda^{4}}+p}
\end{aligned}
$$

общее решение уравнения (1) и граничные условия (2) представим в виде:

$$
\begin{gathered}
w=A \cos \left(\lambda_{1} \xi\right)+B \sin \left(\lambda_{1} \xi\right)+C \operatorname{ch}\left(\lambda_{2} \xi\right)+D \operatorname{sh}\left(\lambda_{2} \xi\right), \\
w_{1}=0, \frac{\partial w}{\partial \xi}=0, \quad(\xi=0,1) .
\end{gathered}
$$

Граничные условия и условия (3) запишем в развернутом виде:

$$
\begin{gathered}
A+C=0 ; B \lambda_{1}+D \lambda_{2}=0 \\
A \cos \lambda_{1}+B \sin \lambda_{1}+C \operatorname{ch} \lambda_{2}+D \operatorname{sh} \lambda_{2}=0 \\
\lambda_{1} \cdot\left(-A \sin \lambda_{1}+B \cos \lambda_{1}\right)+\lambda_{2} \cdot\left(C \operatorname{sh} \lambda_{2}+D \cdot \operatorname{ch} \lambda_{2}\right)=0 .
\end{gathered}
$$

Для того чтобы $A, B, C, D$ не были равны нулю одновременно, необходимо, чтобы определитель основной матрицы был равен нулю. Это условие дает частотное уравнение:

$$
\lambda^{2}\left(1-\cos \lambda_{1} \cdot \operatorname{ch} \lambda_{2}\right)+p \sin \lambda_{1} \cdot \operatorname{sh} \lambda_{2}=0
$$

Таким образом, в приведенной простейшей модели трубопровода фигурируют параметры $p$ и $\lambda$, которые зависят от усилия растяжения в трубе $T$, толщины стенки трубопровода $h$, плотности $\rho_{i}$ и давления $P_{i}$ жидкости внутри трубопровода. Отметим, что при $p=0$ частотное уравнение принимает вид $[12,13]$.

\section{3. Прямая задача}

Расчеты проведены для следующих параметров трубы: $E=2,0 \cdot 10^{11} \mathrm{H} / \mathrm{m}^{2} ; \rho=7800 \mathrm{\kappa} / \mathrm{M}^{3}$; $R_{i}=0,259 \mathrm{м} ; L=25$ м. Получены зависимости первой и второй собственных частот изгибных колебаний трубопровода от осевого усилия $T$ при давлении внутри трубопровода $P_{i}=0$, плотности жидкости в трубопроводе $\rho_{i}=0$ для $h=4,5,6$ мм, из чего следует, что с увеличением осевого усилия $T$ происходит увеличение собственных частот изгибных колебаний трубы. Из анализа зависимостей первой и второй собственных частот изгибных колебаний трубопровода от толщины стенки трубы $h$ при давлении внутри трубопровода $P_{i}=0$, плотности жидкости в трубопроводе $\rho_{i}=0$ при осевом усилии $T=-500 ; 0 ; 500$ кН следует, что с увеличением толщины стенки трубы $h$ происходит уменьшение собственных частот изгибных колебаний трубопровода для растягивающего осевого усилия и увеличение собственных частот изгибных колебаний трубопровода для сжимающего осевого усилия. Полученные зависимости первой и второй собственных частот изгибных колебаний трубопровода от плотности жидкости в трубопроводе $\rho_{i}$ при осевом усилии $T=0$, толщине стенки трубы $h=5$ мм при давлении внутри трубопровода $P_{i}=0 ; 0,5 ; 1,0$ МПа позволяют сделать вывод, что с увеличением плотности 
жидкости в трубопроводе $\rho_{i}$ происходит уменьшение собственных частот изгибных колебаний трубы, а с увеличением давления внутри трубопровода $P_{i}$ происходит уменьшение собственных частот изгибных колебаний трубы.

\section{4. Обратная задача}

Из решения прямой задачи для трубы с вышеприведенными параметрами: $h=5$ мм; $T=0 ; P_{i}=0 ; \rho_{i}=800$ кг $/ \mathrm{M}^{3}$ следует, что первая и вторая собственные частоты трубопровода $f_{1}=2,799$ Гц; $f_{2}=7,717$ Гц. Решение обратной задачи для трубопровода с вышеприведенными параметрами при $f_{1}=2,7$ Гц; $f_{2}=7,7$ Гц дает: $h=5,399$ мм; $T=-479,721$ кН. По двум частотам изгибных колебаний можно определить осевое усилие $T$ и толщину стенки трубы $h$. На рис. 1 представлены зависимости от первой частоты изгибных колебаний $f_{1}$ осевого усилия $T(a)$ и толщины стенки трубы $h(б)$ для различных частот изгибных колебаний $f_{2}$ : 7,70 Гц - кривая $1 ; 7,72$ Гц $-2 ; 7,74$ Гц -3 при $P_{i}=0$ и $\rho_{i}=800$ кг $/ \mathrm{m}^{3}$. По двум частотам изгибных колебаний также можно определить осевое усилие $T$ и плотность жидкости в трубопроводе $\rho_{i}$. На рис. 2 приведены зависимости от первой частоты изгибных колебаний $f_{1}$ осевого усилия $T(a)$ и толщины стенки трубы $h(\sigma)$ для различных частот изгибных колебаний $f_{2}: 7,70$ Гц - кривая $1 ; 7,72$ Гц $-2 ; 7,74$ Гц -3 при $P_{i}=0$ и $h=5$ мм. По двум частотам изгибных колебаний также можно определить плотность жидкости в трубопроводе $\rho_{i}$ и толщину стенки трубы $h$ или давление $P_{i}$ и плотность жидкости в трубопроводе $\rho_{i}$, или давление $P_{i}$ и толщину стенки трубы $h$.
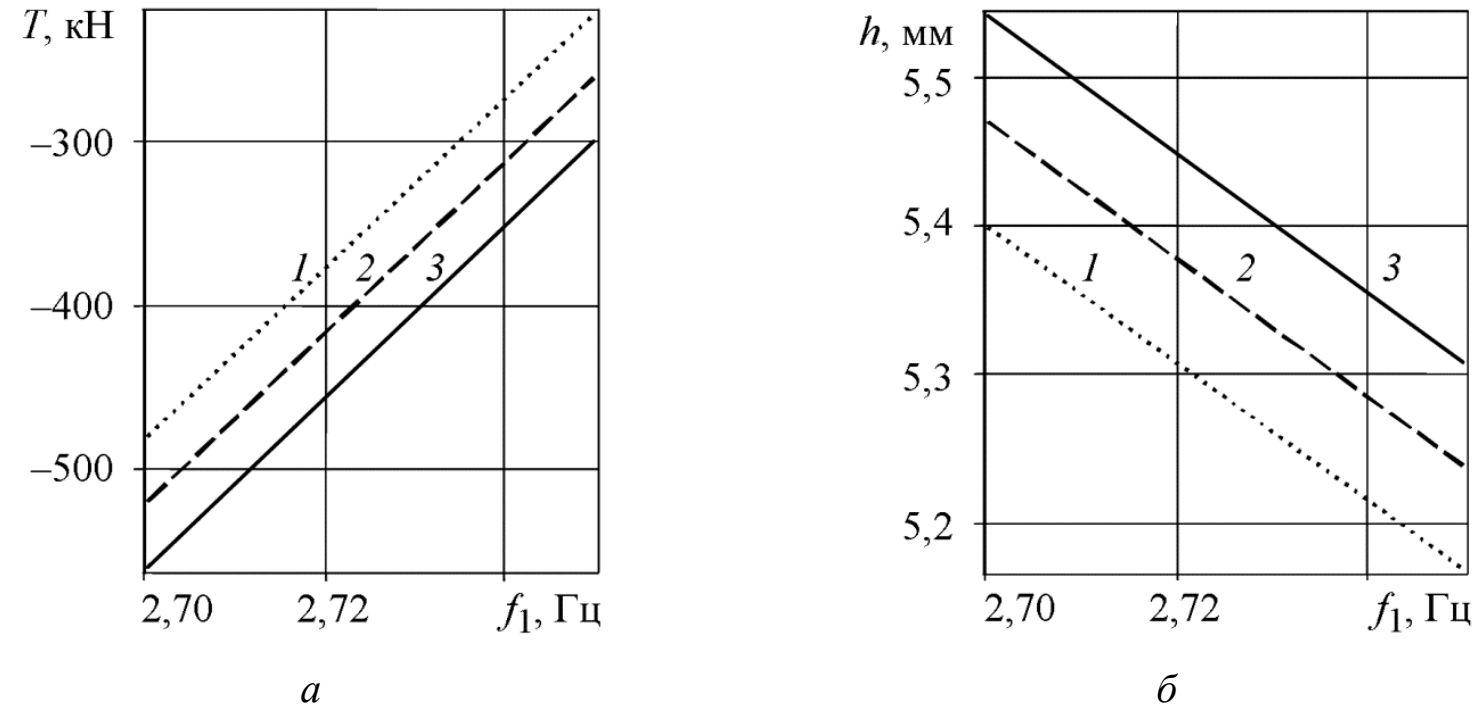

Рис. 1. Зависимости от первой частоты изгибных колебаний $f_{1}$ осевого усилия $T(a)$ и толщины стенки трубы $h(б)$ для различных частот изгибных колебаний $f_{2}$ :

$$
7,70 \text { Гц - кривая } 1 ; 7,72 \text { Гц }-2 ; 7,74 \text { Гц }-3 \text { при } P_{i}=0 \text { и } \rho_{i}=800 \text { кг/м }{ }^{3}
$$

По двум частотам изгибных колебаний также можно определить плотность жидкости в трубопроводе $c_{i}$ и толщину стенки трубы $h$. На рис. 3 приводятся зависимости от первой частоты изгибных колебаний $f_{1}$ плотность жидкости в трубопроводе $\rho_{i}(a)$ и толщины стенки трубы $h\left(\right.$ б) для различных частот изгибных колебаний $f_{2}: 7,700$ Гц - кривая $1 ; 7,702$ Гц - 2; 7,704 Гц -3 при $P_{i}=0,1$ МПа и $T=0$. 


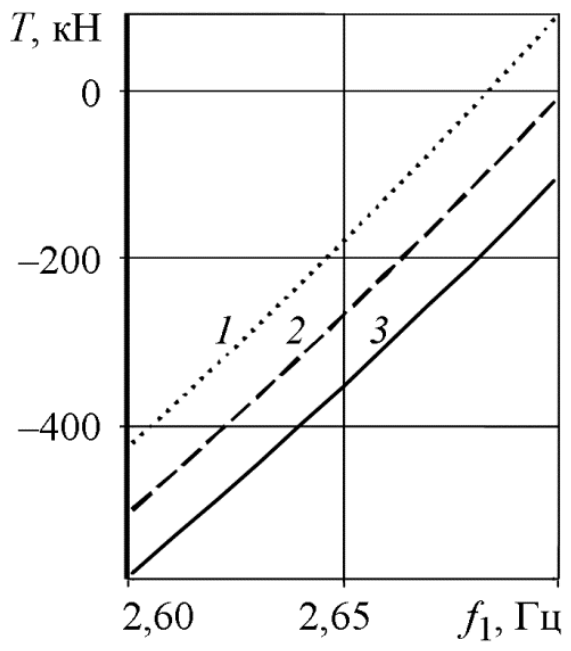

$a$

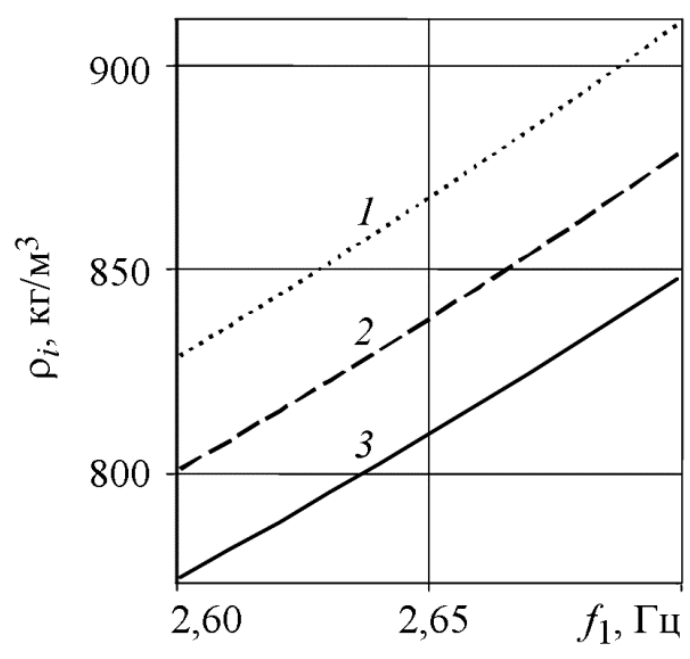

$\sigma$

Рис. 2. Зависимости от первой частоты изгибных колебаний $f_{1}$ осевого усилия $T(a)$ и плотности жидкости в трубопроводе $\rho_{i}(б)$ для различных частот изгибных колебаний $f_{2}$ :

7,40 Гц - кривая $1 ; 7,45$ Гц $-2 ; 7,50$ Гц -3 при $P_{i}=0$ и $h=5$ мм

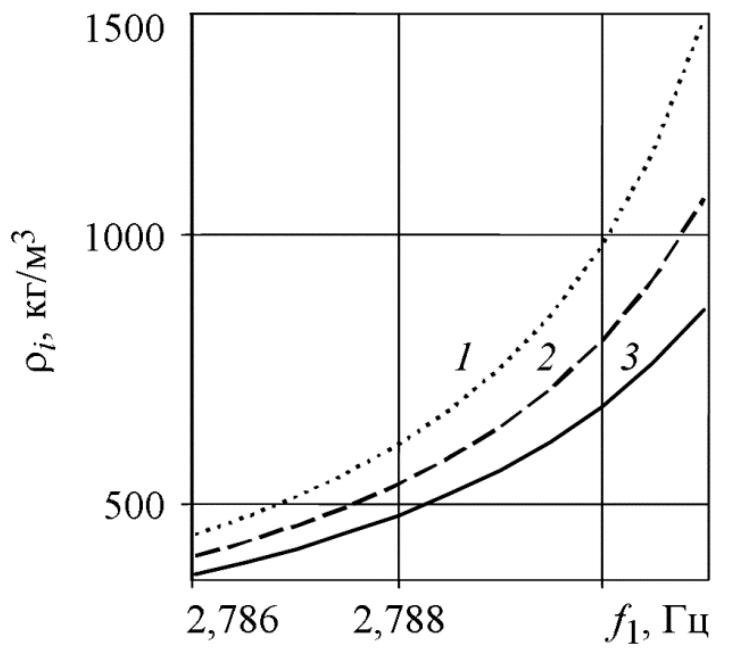

$a$

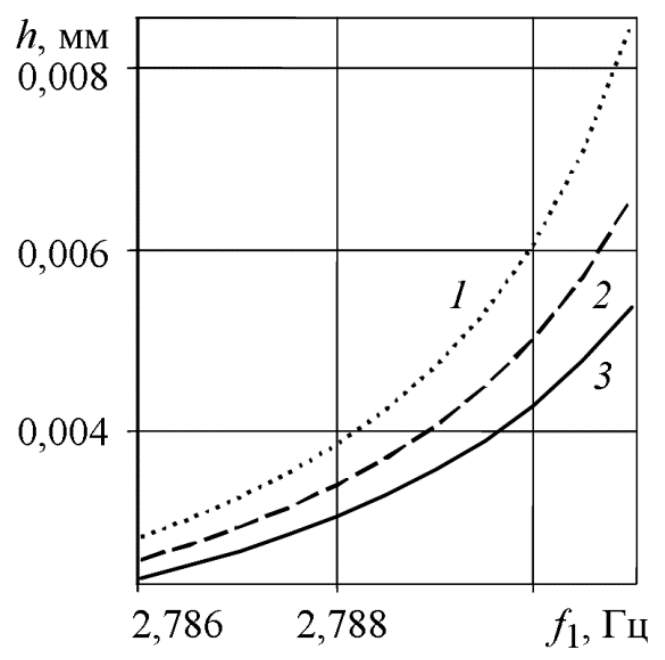

$\sigma$

Рис. 3. Зависимости от первой частоты изгибных колебаний $f_{1}$ плотность жидкости в трубопроводе $\rho_{i}(a)$ и толщины стенки трубы $h(\sigma)$ для различных частот изгибных колебаний $f_{2}:$ 7,700 Гц - кривая $1 ; 7,702$ Гц - 2; 7,704 Гц - 3 при $P_{i}=0,1$ МПа и $T=0$

По двум частотам изгибных колебаний также можно определить давление $P_{i}$ и плотность жидкости в трубопроводе $\rho_{i}$. На рис. 4 представлены зависимости от первой частоты изгибных колебаний $f_{1}$ давления $P_{i}(a)$ и плотности жидкости в трубопроводе $\rho_{i}(\sigma)$ для различных частот изгибных колебаний $f_{2}: 7,70$ Гц - кривая $1 ; 7,71$ Гц $-2 ; 7,72$ Гц -3 при $h=5$ мм и $T=0$.

По двум частотам изгибных колебаний также можно определить давление $P_{i}$ и толщину стенки трубы $h$. На рис. 5 представлены зависимости от первой частоты изгибных колебаний $f_{1}$ давления $P_{i}(a)$ и толщины стенки трубы $h(\sigma)$ для различных частот изгибных

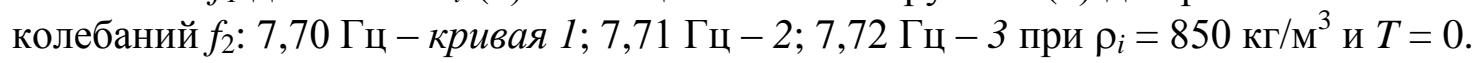




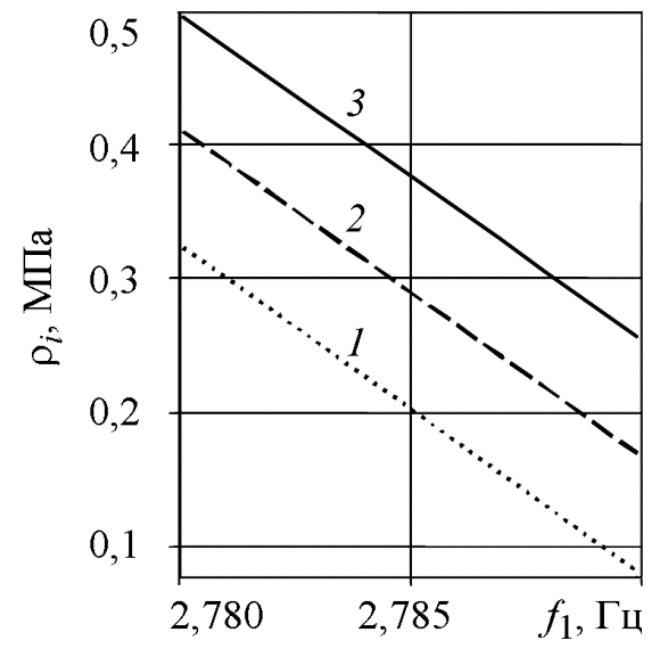

$a$

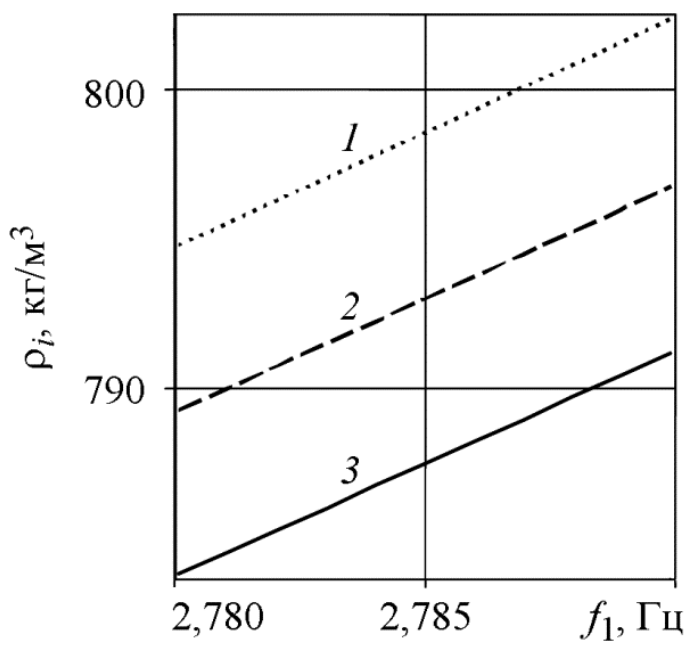

$\sigma$

Рис. 4. Зависимости от первой частоты изгибных колебаний $f_{1}$ давления $P_{i}(a)$ и плотности жидкости в трубопроводе $\rho_{i}(б)$ для различных частот изгибных колебаний $f_{2}$ : 7,70 Гц - кривая $1 ; 7,71$ Гц $-2 ; 7,72$ Гц -3 при $h=5$ мм и $T=0$

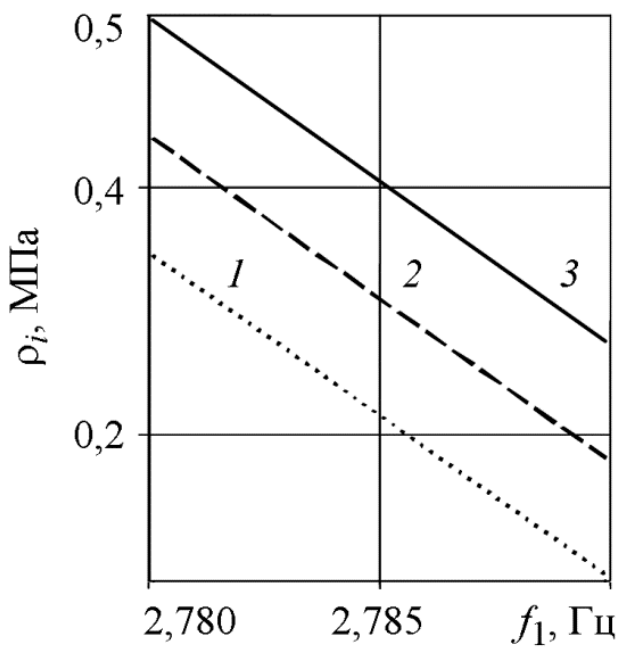

$a$

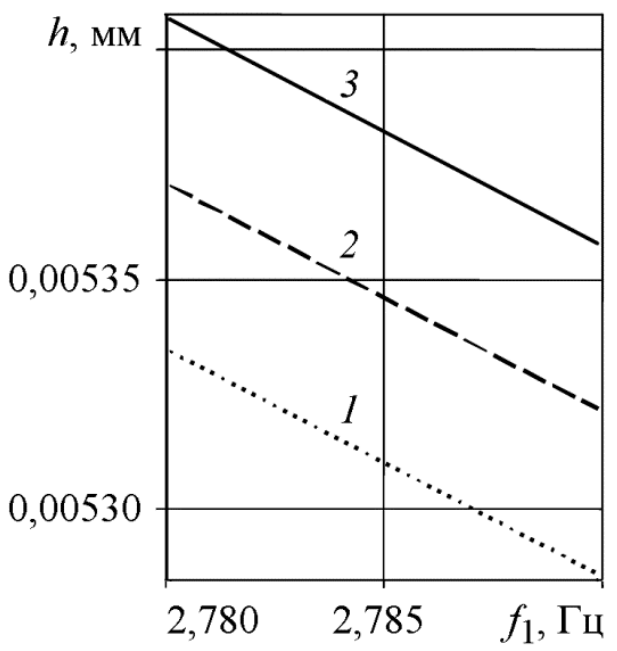

$\sigma$

Рис. 5. Зависимости от первой частоты изгибных колебаний $f_{1}$ давления $P_{i}(a)$ и толщины стенки трубы $h(б)$ для различных частот изгибных колебаний $f_{2}$ :

7,70 Гц - кривая $1 ; 7,71$ Гц $-2 ; 7,72$ Гц -3 при $\rho_{i}=850$ кг/м ${ }^{3}$, и $T=0$

\section{5. Заключение}

Установлено, что с увеличением осевого усилия происходит увеличение собственных частот изгибных колебаний трубы, а с увеличением толщины стенки трубы происходит уменьшение собственных частот изгибных колебаний трубы для растягивающего осевого усилия и увеличение собственных частот изгибных колебаний трубы для сжимающего осевого усилия. Получено, что с увеличением плотности жидкости или давления внутри трубопровода происходит уменьшение собственных частот изгибных колебаний трубы.

По двум собственным частотам изгибных колебаний трубы можно определить осевое усилие и толщину стенки трубы или осевое усилие и плотность жидкости в трубопроводе, или плотность жидкости в трубопроводе и толщину стенки трубы, или давление и плотность жидкости в трубопроводе, или давление в трубопроводе и толщину его стен- 
ки. Результаты работы могут быть применены для определения осевого усилия и толщины стенки трубы, или осевого усилия и плотности жидкости в трубопроводе, или плотности жидкости в трубопроводе и толщины стенки трубы, или давления и плотности жидкости в трубопроводе, или давления в трубопроводе и толщины его стенки по двум собственным частотам изгибных колебаний.

\section{Благодарность}

Работа поддержана средствами государственного бюджета по госзаданию (№ 0246-2019-0088) и грантом РФФИ (№ 18-01-00150).

\section{Литература}

1. Сидоров Б. В., Мартынов С. А. Рекомендуемая технология диагностики подземных трубопроводов // Контроль. Диагностика. - 2005. - № 12. - С. 18-19.

2. Gladwell G. M. L. Inverse problems in vibration. - Dordrecht, Boston, London : Kluver Academic Publishers, 2004. - (Русский перевод: Глэдвелл Г.М.Л. Обратные задачи теории колебаний. - М.-Ижевск : НИЦ «Регулярная и хаотическая динамика», 2008. - 608 с.).

3. Guangming Dong and Jin Chen. Vibration analysis and crack identification of a rotor with open cracks // Japan Journal of Industrial and Applied Mathematics. - 2011. - Vol. 28, no. 1. P. 171-182. - DOI: 10.1007/s13160-011-0031-3.

4. Интегральный диагностический признак идентификации повреждений в элементах стержневых конструкций / В. А. Акопьян, А. В. Черпаков, Е. В. Рожков, А. Н. Соловьев // Контроль. Диагностика. - 2012. - № 7. - С. 50-56.

5. Yunwei Zhang, Guozheng Yan. Detection of gas pipe wall thickness based on electromagnetic flux leakage // Russian Journal of Nondestructive Testing. - 2007. - Vol. 43, iss. 2. P. 123-132. - DOI: 10.1134/S1061830907020088.

6. Куликов В. В. Толщина стенки трубы, нагруженной давлением // Строительство нефтяных и газовых скважин на суше и на море. -2009 . - № 7. - С. 6-8.

7. Kucheryavyi V. I., Mil'kov S. N. Reliability analysis of a compression section of a gas pipeline with the presence of longitudinal cracks // Journal of Machinery Manufacture and Reliability. 2011. - Vol. 40, no. 3. - P. 290-293. - DOI: 10.3103/S1052618811030095.

8. Novgorodov D. V., Rybalko V. G., Shleyenkov A. S. The stress state instability factor and its effect on the growth of stress corrosion cracking defects // Diagnostics, Resource and Mechanics of materials and structures. - 2018. - Iss. 6. - P. 249-254. - DOI: 10.17804/2410-9908.2018.6.249-254.

9. Novgorodov D. V., Rybalko V. G., Shleyenkov A. S., and Surkov A. Yu. A study of gas pipeline emergency failure // Diagnostics, Resource and Mechanics of materials and structures. 2018. - Iss. 6. - P. 237-248. - DOI: 10.17804/2410-9908.2018.6.237-248.

10. Chirikov V. A., Dimitrov D. M., Kostov K. P. Universal experimental relation for natural friquencies of transversal vibration of stubby free-free beams // Diagnostics, Resource and Mechanics of materials and structures - 2015. - Iss. 4. - P. 42-51. - DOI: 10.17804/2410-9908.2015.4.042-051.

11. Хакимов А. Г. Определение плотности жидкости и внутреннего давления в трубопроводе по собственным частотам изгибных колебаний // Проблемы сбора, подготовки и транспорта нефти и нефтепродуктов. - 2014. - № 1. - С. 37-43.

12. Прочность, устойчивость, колебания : справочник в трех томах. Том 3 / под общ. ред. И. А. Биргера и Я. Г. Пановко. - М. : Машиностроение, 1968. - 567 с.

13. Вибрации в технике : справочник в 6 томах. Том 1 : Колебания линейных систем / под ред. В. В. Болотина. - М. : Машиностроение, 1999. - 482 с. 